\title{
A Classical View of Quantum Time Crystals
}

\author{
Numerical studies indicate that certain types of time crystals might be \\ described using classical physics - a result that could vastly simplify the \\ theoretical description of these systems.
}

\author{
By Georg Engelhardt
}

W

hen a collection of atoms reaches a sufficiently low temperature, they arrange themselves periodically in space to form a crystal. Since the equations that describe the atoms' motion do not contain any information that prescribes a crystal lattice, this phase transition isn't trivial to explain even in its simplest form. Experimental and theoretical studies have revealed many types of classical and quantum phase transitions that lead to spatially ordered states. But there are other possible forms of order beyond that of the atoms' spatial arrangement. In 2012, Frank Wilczek theorized a novel type of phase transition in which matter spontaneously exhibits time-periodic motion, meaning that it arranges itself periodically in time rather than in space (Fig. 1). The resulting phase of matter is called a quantum time crystal (see Viewpoint: Crystals of Time).

After realizing that time crystals cannot arise under equilibrium conditions, as Wilczek originally proposed [1], many researchers assumed that time crystals are a genuine nonequilibrium quantum effect. However, by employing large-scale numerical simulations, two independent collaborations led by teams at the University of Cambridge, UK, and at the University of California, Berkeley, have found that certain types of time crystals, known as prethermal, can be described using purely classical equations of motion. Their simulations show that many-body systems of classical spins exhibit all the basic features of quantum time crystals, indicating that prethermal time crystals are a classical effect [2-4]. The finding suggests that a description rooted in classical physics can suffice to describe these time crystals and may suggest ways to create them experimentally.
Because of the nonequilibrium requirement, researchers have focused the search for time crystals on quantum many-body systems that are driven by an external field that's periodic in time. This dynamic state of matter, dubbed discrete time crystals [5-7], has opened new experimental realization possibilities in, for example, nuclear spins, trapped ions, or cold atoms (see Viewpoint: How to Create a Time Crystal and Viewpoint: Time Crystals in Open Systems). In a discrete time crystal, the system's arrangement oscillates with a time period $\tau$, which is an integer multiple of the driving period $T(\tau=n T$, where $n$ is the "order" of the time crystal).

Simulations in one-dimensional quantum systems have shown that the creation of a stable time crystal requires long-range interactions between the system constituents (e.g., spins or atoms). But long-range interactions are difficult to realize and control experimentally; and exploring their influence theoretically remains difficult because of limitations in the ability to account for such interactions in the simulation of quantum systems.

Another challenge to studying time crystals is the fact the periodic driving of a many-body system causes the system to heat up, eventually reaching a temperature that would destroy both spatial and temporal crystalline order. This fate can be evaded through various approaches. One popular approach involves a so-called prethermal state in which, according to quantum theory, a periodically driven system behaves like an undriven system for extremely long times until thermalization sets in [8-10]. However, a full theoretical picture for describing discrete time crystals in this state has not yet been developed. 


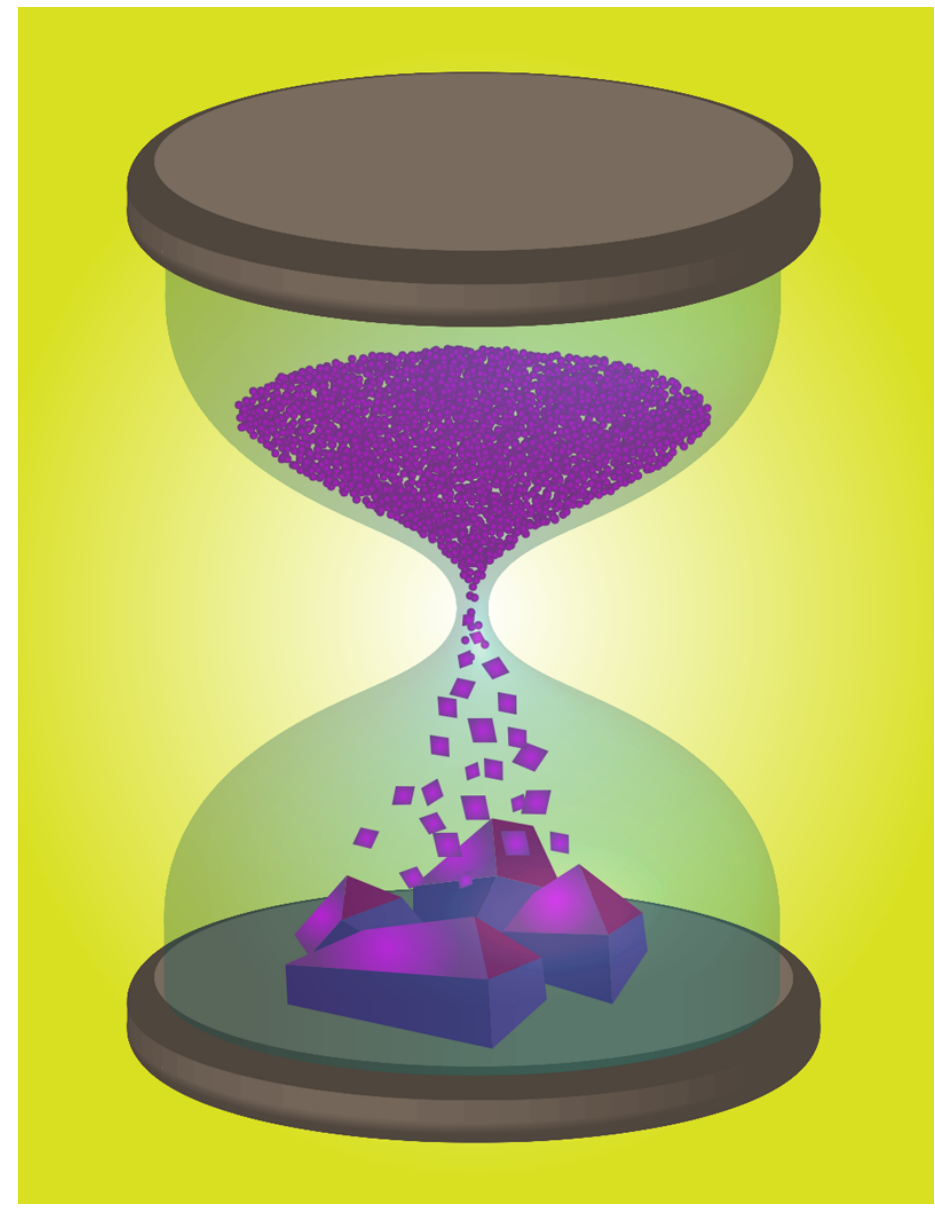

Figure 1: A description based on classical physics serves to describe the quantum phenomena known as time crystals. Time crystals arise when matter spontaneously forms a pattern in time, much as a normal crystal arises when matter spontaneously forms a pattern in space.

Credit: G. Engelhardt; APS/Alan Stonebraker

The two research groups now report simulations that show striking similarities between quantum and classical dynamics in a prethermal state [2-4]. The results have twofold implications. First, it may simplify the simulation of prethermal time crystals because of the numerical advantage in simulating classical systems over quantum systems. Second, the analogy between the quantum and the classical effect indicates that prethermal time crystals arise not because of, but in spite of, the quantum fluctuations that had been considered necessary to stabilize the prethermal phase and the discrete time crystal.

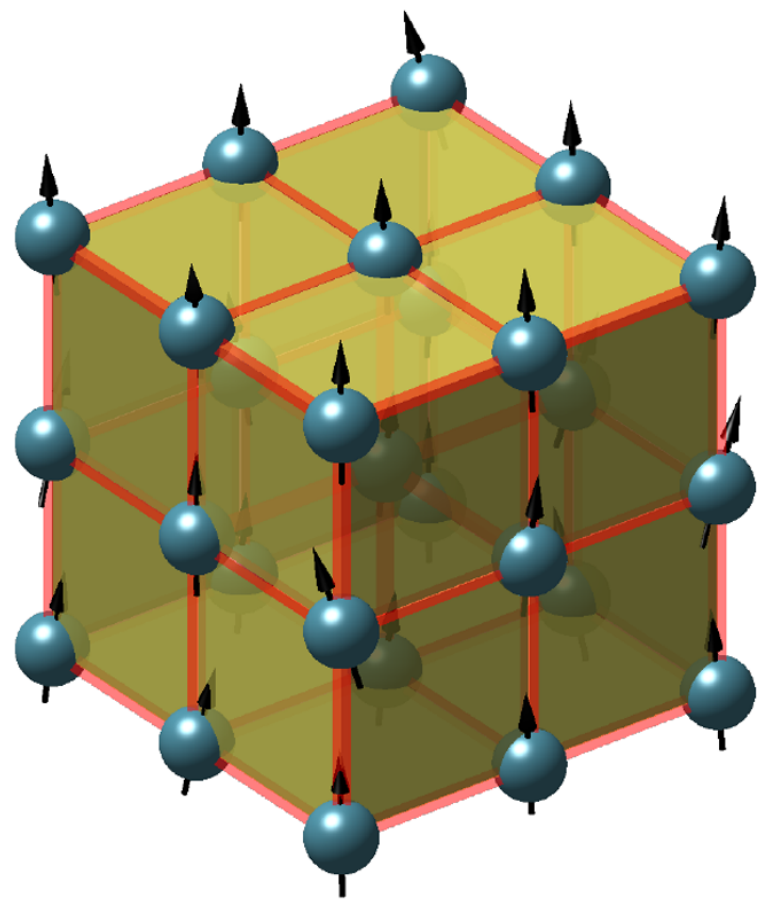

Figure 2: Numerical simulations of classical spins (blue spheres) arranged in this three-dimensional lattice show that time crystals may arise and remain stable in a system where each spin interacts only via short-range interactions (red line segments) with other nearby spins.

Credit: A. Pizzi et al. [2]; adapted by APS/Alan Stonebraker

Both research groups investigated periodically driven classical lattices of interacting spins (Fig. 2), which serve as an analog to commonly investigated quantum spin chains-for example, qubits on a quantum processor. The teams independently predicted a long-lasting prethermal phase in close correspondence with the spins' quantum counterparts. During the prethermal phase, the spin system could feature long-lasting discrete time crystals with a high and even fractional (for example, $n=7 / 20$ ) order- an effect that is much harder to stabilize than the common time crystal with integer $n$. Both groups found that short-range interactions-by which each spin interacts only with nearby spins-are sufficient for time crystals to remain stable in two or three dimensions. This offers the advantage that short-range interactions can more easily be experimentally controlled than long-range 
interactions. Compared to the vast computational power that would be required to solve the Schrödinger equation, the simulation involving classical mechanics is more feasible.

The simulations by Pizzi and his colleagues demonstrated that in a periodically driven 1D spin lattice, classical and quantum observable quantities exhibit a one-to-one correspondence [2, $3]$. For example, the time dependence of the mean energy and magnetization of the classical spin system share the same features as their quantum counterparts. Intriguingly, the time evolution of a genuine quantum property called entanglement entropy is mirrored by a quantity that measures the difference of the time evolution of two states, whose initial states had been very similar. This quantum-classical correspondence allowed the authors to investigate time crystals by carrying out classical simulations. As a result, they could probe the parameter space in two and three dimensions and predict that the time crystals are more robust in a higher dimension.

Ye and his colleagues questioned the role of effective time-independent Hamiltonians in quantum and classical systems [4]. In a quantum system, an effective Hamiltonian can approximately reproduce the time dynamics of the microscopic states of the system. But the same is not generally true for classical time evolution because the emergence of chaotic dynamics makes the system unpredictable. However, the researchers' Monte Carlo simulations of the classical spin lattice showed the system is still sufficiently predictable that an effective Hamiltonian can predict the probably distribution of expectation values.

The correspondence of classical and quantum time crystals in a spin system's prethermal state opens new research directions. As a next step, it must be clarified whether similar conclusions are applicable to other types of time crystals that are stabilized by physical effects other than the prethermal state. Other proposed stabilization mechanisms include, for instance, harnessing many-body localization to mitigate the heating associated with the external driving. Do these time crystals also have a classical counterpart or are they purely quantum effects?
Furthermore, the continued progress of quantum processors will boost the power of quantum simulation, allowing detailed tests of whether quantum systems truly behave as the classical counterparts that Pizzi and Ye and their respective colleagues have identified [11]. What's more, the newly identified classical correspondence might guide researchers in developing a simple theoretical picture of time crystals, ultimately allowing them to predict and control time-crystalline behavior in diverse experimental platforms.

Georg Engelhardt: Beijing Computational Science Research Center, Beijing, China

\section{REFERENCES}

1. H. Watanabe and Masaki Oshikawa, "Absence of quantum time crystals," Phys. Rev. Lett. 114, 251603 (2015).

2. A. Pizzi et al., "Classical prethermal phases of matter," Phys. Rev. Lett. 127, 140602 (2021).

3. A. Pizzi et al., "Classical approaches to prethermal discrete time crystals in one, two, and three dimensions," Phys. Rev. B 104, 094308 (2021).

4. B. Ye et al., "Floquet phases of matter via classical prethermalization," Phys. Rev. Lett. 127, 140603 (2021).

5. D. V. Else et al., "Floquet time crystals," Phys. Rev. Lett. 117, 090402 (2016).

6. V. Khemani et al., "Phase structure of driven quantum systems," Phys. Rev. Lett. 116, 250401 (2016).

7. N. Y. Yao et al., "Discrete time crystals: Rigidity, criticality, and realizations," Phys. Rev. Lett. 118, 030401 (2017).

8. D. A. Abanin et al., "Effective Hamiltonians, prethermalization, and slow energy absorption in periodically driven many-body systems," Phys. Rev. B 95, 014112 (2017).

9. E. Canovi et al., "Stroboscopic prethermalization in weakly interacting periodically driven systems," Phys. Rev. E 93, 012130 (2016).

10. T. Mori et al., "Rigorous bound on energy absorption and generic relaxation in periodically driven quantum systems," Phys. Rev. Lett. 116, 120401 (2016).

11. X. Mi et al., "Observation of time-crystalline eigenstate order on a quantum processor," arXiv:2107.13571. 